\title{
A New Population of Planetary Nebulae Discovered in the LMC
}

\author{
Warren A. Reid ${ }^{1} \dagger$ and Quentin A. Parker ${ }^{1,2}$ \\ ${ }^{1}$ Department of Physics, Macquarie University, Sydney, NSW, 2109, Australia \\ email: warren@ics.mq.edu.au \\ ${ }^{2}$ Anglo-Australian Observatory, Sydney, NSW, 2102 Australia, \\ email: qap@ics.mq.edu.au
}

\begin{abstract}
We report our discovery of 471 planetary nebulae (PNe) in the central $25 \mathrm{deg}^{2} \mathrm{re}^{-}$ gion of the Large Magellanic Cloud (LMC) following confirmatory two-degree Field (2dF) spectroscopy on the Anglo-Australian Telescope (AAT). Candidate emission sources were discovered using deep, high resolution UKST stacked Short Red (SR) and $\mathrm{H} \alpha$ images which go deeper than any previously available. The two digitized stacks were then merged to reveal emission sources. The new PNe have implications for the LMC PN luminosity function, kinematics, abundance gradients, chemical evolution and the initial to final mass relation for low to intermediate mass stars via the AGB halos revealed on the $\mathrm{H} \alpha$ map. Excitation classes are presented.
\end{abstract}

Keywords. Magellanic Clouds, Surveys, Planetary Nebulae

\section{Introduction}

The study of planetary nebulae (PN), including the determination of their physical properties and luminosity function (PNLF), has been a difficult task within our galaxy due to the inherent problems of accurate distance determination. This is a problem which can be overcome by studying PNe in a nearby galaxy such as the Large Magellanic Cloud (LMC) where, by approximation, all PN can be thought of as residing at the same $50 \mathrm{kpc}$ distance. Several studies have been undertaken to survey the LMC for PNe but the total number of confirmed PNe has remained modest $(\sim 300)$ compared to the several thousand that might be expected (Jacoby 1980). This has limited the ability to provide precise estimates of the PNLF and other key parameters.

A high resolution AAO/UKST H $\alpha$ project was initiated by Parker \& Morgan to obtain multiple exposures and probe deeper into the LMC. Twelve well-matched 2-hour H $\alpha$ exposures and six 15-min equivalent Short-Red (SR) exposures of the LMC field were stacked by SuperCOSMOS, creating 10-micron ( 0.67 arcsec) pixel data which goes 1.35 and 1 magnitudes deeper than individual exposures as expected giving R.equiv $\sim 22$ for $\mathrm{H} \alpha$ and $\sim 21.5$ for SR. The stacked data sets were registered onto the same pixel grid followed by accurate assignment of a WCS creating the most uniform narrow-band map of the LMC ever made. Candidate emission sources were found using an adaptation of a technique available within the ATNF KARMA software package. Careful selection of software parameters allowed the intensity of the matched $\mathrm{H} \alpha$ and SR FITS images to be perfectly balanced allowing only peculiarities of one or other pass-band to be observed and measured (Reid \& Parker, 2006). The veracity of the technique has been powerfully demonstrated firstly by the ease of independent re-identification of all previously known $\mathrm{PNe}$ and secondly by the spectroscopic confirmation of 471 new LMC PNe.

$\dagger$ Present address: Macquarie University, Sydney, NSW, 2109, Australia 


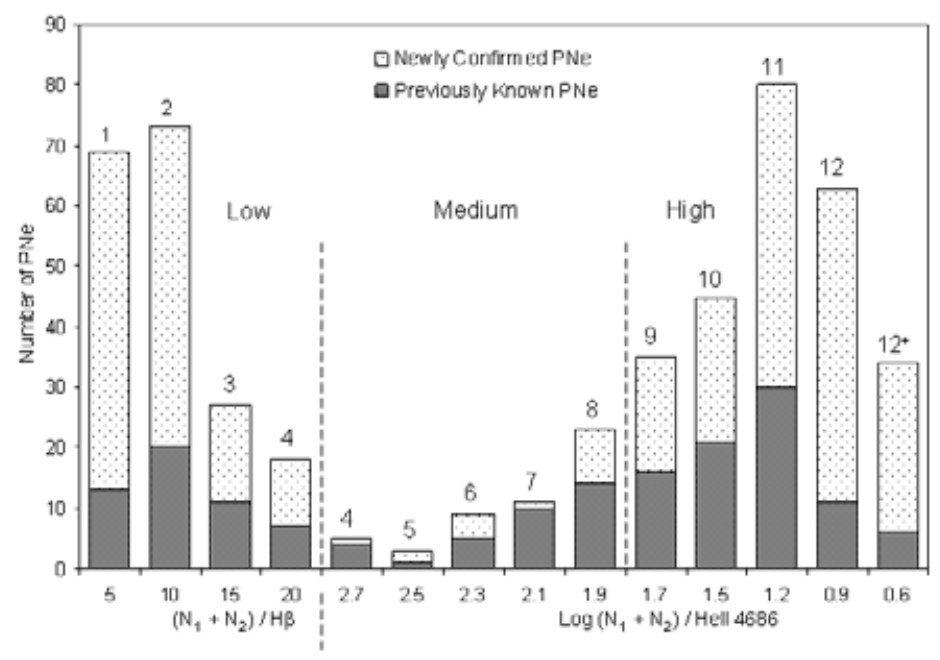

Figure 1. Graphic representation of the excitation class $(\rho)$ for previously known and newly confirmed PNe across the whole LMC survey area. Classification is dependent upon $\left(\mathrm{N}_{1}+\right.$ $\left.\mathrm{N}_{2}\right) / \mathrm{H} \beta$ for low excitation PNe $(\rho=1-4)$ and $\log \left(\mathrm{N}_{1}+\mathrm{N}_{2}\right) / \mathrm{He}$ II 4686 for medium $(\rho=4-8)$ and high excitation $\mathrm{PNe}\left(\rho=9-12^{+}\right)$.

\section{Complete Follow-up Optical Spectroscopy}

Spectral follow-up of candidates was completed in February 2005 yielding a complete, magnitude-limited sample of several thousand new emission sources across the entire LMC main-bar field. Confirmatory spectroscopy was performed using $2 \mathrm{dF}$ on the AAT, the 1.9-m telescope at SAAO, the 2.3-m Advanced Technology Telescope at the MSSSO and FLAMES on the ESO VLT2. Optical spectroscopy not only allowed us to identify $\mathrm{PNe}$ from our large sample but results are allowing physical conditions to be determined.

\section{Excitation Classes}

Excitation classes have been derived for all LMC PNe in our survey area, the new PNe showing similar trends to previously known PNe (Figure 1). Columns 1-4 are low excitation PNe, columns 4-8 are medium excitation and columns 9-12+ are high excitation based on the identification of He II 4686. Ratios are given on the $x$-axis and the number of PNe in each bin is specified on the $y$-axis. The system is based on the ratio of $I\left(\mathrm{~N}_{1}+\right.$ $\mathrm{N}_{2}$ ) /I(He II 4686) or $\mathrm{H} \beta$ emission line intensities. In the full LMC sample, this ratio varies from 1 (equivalent to $2 \sigma$ ) to 500 . The excitation class $\rho$ is subdivided by integer numbers from 4 to $12+$ according to $\log I\left(\mathrm{~N}_{1}+\mathrm{N}_{2}\right) / I(\mathrm{He}$ II 4686$)$.

For each excitation class, the logarithm of the excitation class corresponds to the limits \pm 0.1 . The highest excited PNe are placed into the $12+$ category. They have a mean logarithmic value equal to 0.6 relating to nucleus temperatures of $300,000 \mathrm{~K}$ and higher. Where the He II 4686 line is extremely low or missing in low excitation PNe $(\rho=1-4)$ the line ratio $I\left(\mathrm{~N}_{1}+\mathrm{N}_{2}\right) / I\left(\mathrm{H}_{\beta}\right)$ is used. This ratio is linearly sensitive to the $\mathrm{O}^{++} / \mathrm{O}$ ratio (much like $\mathrm{He}^{+} / \mathrm{He}$ ) and exponentially to the electron temperature $T_{e}$.

\section{References}

Jacoby G.H. 1980 AJ Suppl. Series, 42, 1

Reid W.A. and Parker Q.A., 2006, MNRAS, 365, 401R 